\title{
The ebbing tide of the reservoir-wave model
}

\author{
Jonathan P. MYNARD ${ }^{a, b}$, Joseph J. SMOLICH ${ }^{a, b}$, Alberto AVOLIO \\ ${ }^{a}$ Heart Research, Clinical Sciences, Murdoch Childrens Research Institute, Melbourne, \\ Australia \\ ${ }^{\mathrm{b}}$ Department of Paediatrics, University of Melbourne, Melbourne, Australia \\ ${ }^{c}$ Australian School of Advanced Medicine, Macquarie University, Sydney, Australia
}

Brief title: The ebbing of the reservoir-wave model

Editorial commentary relating to:

Borlotti, A., C. Park, K. H. Parker and A. W. Khir. "Reservoir and reservoir-less pressure effects on arterial waves in the canine aorta." Journal of Hypertension. DOI: 10.1097/HJH.0000000000000425

Segers, P., L. Taelman, J. Degroote, J. Bols and J. Vierendeels. "The aortic reservoir-wave as a paradigm for arterial haemodynamics: insights from three-dimensional fluid-structure interaction simulations in a model of aortic coarctation." Journal of Hypertension. DOI: 10.1097/HJH.0000000000000449.

Corresponding Author:

Jonathan P. Mynard

E-mail: jonathan.mynard@mcri.edu.au

Heart Research, Clinical Sciences, Murdoch Childrens Research Institute

50 Flemington Rd. Parkville, VIC, 3052, Australia

Phone: +1 399366038

No conflicts of interest.

Word count (inc. references): 2805

Number of figures: 1

Number of tables: 0 
Models form the foundation on which our language, understanding and quantification of haemodynamics and vascular physiology are built. One of the most well-known models of arterial haemodynamics is the two-element windkessel, which defines our concepts of vascular resistance and compliance, and provides an intuitive explanation of the diastolic pressure decay [1]. An extension to this model, the three-element windkessel, also includes characteristic impedance and has highlighted the important haemodynamic role of aortic size and stiffness on systolic haemodynamics [2]. Similarly, wave propagation models undergird concepts such as pulse wave velocity and wave reflection [3]. Although theoretical debates about models may sometimes appear purely academic, these modelbased concepts and associated metrics are integral to recent and ongoing clinical studies relating to hypertension, vascular aging and other cardiovascular disease [4-9].

The robustness and explanatory power of the model(s) we adopt are therefore of utmost importance, and scrutiny should be applied before a new model is accepted. Questions should be asked of a new model. Is it internally consistent? Is it based on a solid foundation of physics and mathematics? Does it have superior explanatory power than previous models? Can its validity be checked against a gold standard in vivo? If it is a simplified model, can its validity also be checked against a more comprehensive model? Does the model lead to an improved understanding of cardiovascular function, and so provide added value to existing conceptual frameworks?

Questions such as these have been debated recently in relation to the reservoirwave model, originally proposed by Wang et al in 2003 [10], that combines concepts from windkessel and wave propagation models. The key idea in the reservoir-wave model is to separate blood pressure into two components, a wave-independent 'reservoir pressure' 
$\left(P_{\text {res }}\right)$ caused by the filling and emptying of arteries over the cardiac cycle, and an 'excess pressure' $\left(P_{e x}\right)$ related to the propagation and reflection of pressure/flow waves. Two major practical implications of adopting this model are as follows. First, the amplitude of the reservoir pressure would be used to quantify the buffering capacity of the arterial system. Second, wave reflection would be quantified on the basis of $\mathrm{P}_{\mathrm{ex}}$ rather than raw measured pressure. Before adopting this model, however, its key assumptions should be scrutinized.

A major assumption in the reservoir-wave model is that $P_{\text {res }}$ closely tracks the volume of the arterial reservoir, with any change in arterial volume causing a proportional change in $\mathrm{P}_{\text {res. }}$ In this issue Segers et al [11] evaluated this core assumption using a threedimensional computational model of the aorta, incorporating a fluid-structure interaction technique that realistically accounts for blood flow dynamics as well as the spatial and temporal motion of the aortic wall during the cardiac cycle. Four conditions were tested: normal, mild residual coarctation, stented coarctation and severe re-coarctation. The nonlinear pressure-volume relation of the virtual aorta in the absence of flow and waves was established using static loading over the range 75 to $225 \mathrm{mmHg}$, and then compared with the dynamic relation between $P_{\text {res }}$ and aortic volume $\left(V_{a o}\right)$ during a simulated heart beat (Figure 6 of [11]). In all cases, a marked hysteresis in the $P_{r e s}-V_{a o}$ relation was apparent, which appears to invalidate the assumption that $P_{\text {res }}$ bears a simple relationship to $V_{a o}$. Interestingly, the clockwise trajectory of the $\mathrm{P}_{\text {res }}-\mathrm{V}_{\text {ao }}$ loop implied that work is being done on the fluid by the aorta, which is clearly an implausible characteristic of $\mathrm{P}_{\text {res, }}$, but is consistent with data in dogs showing that aortic volume (estimated as the difference between proximal inflow and distal outflow volumes derived from flow probes) leads $\mathrm{P}_{\text {res }}$ in early systole [10]. 
The findings of Segers et al [11] extend previous work highlighting problems with $P_{\text {res. }}$ For instance, it has been shown that the 'wave-independent' $P_{\text {res }}$ propagates along the aorta and fulfils the universally held definition of a wave as a propagating disturbance [12]. Moreover, the calculated resistance and compliance values used to derive $P_{\text {res }}$ do not represent true arterial resistance and compliance, but exhibit errors of up to $50 \%$ that are directly dependent on wave effects [13]. While evidence to support the assertion that $P_{\text {res }}$ is "fundamentally different" to a wave [14] is lacking, various lines of evidence indicate that $P_{\text {res }}$ is profoundly influenced by, and even determined by, waves. Thus, it was recently shown that the reservoir behaviour of the arterial system can be explained solely in terms of waves, and that the windkessel is simply an approximation of more complex wave phenomena [15]. This wave-based interpretation represents the filling and emptying of the arterial reservoir in terms of 'wave potential', which is fully compatible with conventional wave separation. This new approach not only addresses the perceived problems with wave separation that the reservoir-wave model was designed to solve, but also avoids a number of pitfalls inherent in the classical windkessel (and reservoir-wave) model, such as the assumption of an infinite wave speed and difficulties in estimating windkessel parameters. Furthermore, the magnitude of $\mathrm{P}_{\text {res }}$ is almost identical to twice the conventional backward component of pressure $[16,17]$, suggesting that $P_{\text {res }}$ adds no new quantitative information to currently available indices.

The data presented by Segers et al "implies that the use of excess pressure (rather than total pressure) for the analysis of wave dynamics is intrinsically flawed and doomed to lead to erroneous interpretations of wave dynamics" [11]. A similar conclusion was previously reached by Mynard et al $[12,13,18]$, who compared traditional and reservoirwave approaches to wave analysis using data from one-dimensional blood flow simulations 
in which known reflection sites were prescribed a priori. A number of problems were identified with the $P_{e x}$-based wave analysis, including physically impossible dependencies of early systolic waves on distal reflection sites. However, the most obvious problem was that backward compression waves, arising from sites where characteristic impedance increases, were underestimated or entirely missed by the $\mathrm{P}_{\mathrm{ex}}$-based analysis, whereas the traditional approach accurately quantified the wave reflection. Proponents of the reservoir-wave model have questioned these results by claiming that the numerical simulations were not representative of arterial haemodynamics [14], however this argument lacked substance [18]. It was also argued that the concept of "validity" is difficult to apply in experimental research, since this implies the availability of a gold standard [19].

The need for an in vivo gold standard is also raised by the manuscript of Borlotti et al [20] in this issue, which compared conventional and $P_{e x}$-based versions of wave intensity analysis in dogs at baseline and after occlusion of the aorta at different locations. Their results are very similar to those found previously in sheep [12] and in the aforementioned one-dimensional simulation models $[12,13,18]$, in that backward compression waves were markedly smaller with the reservoir-wave approach. With occlusion of the descending thoracic aorta, the reflection index $(R I$, defined as the ratio of the peak intensities of forward and backward waves) calculated with the reservoir-wave approach was substantially lower than that calculated with the conventional approach $(0.07 \pm 0.04$ versus $0.25 \pm 0.15, P<$ 0.001 , Table 2 in [20]).

The finding that the backward wave was only one quarter (or less) the size of the forward wave in the presence of a large, relatively proximal reflection site due to occlusion may at first appear surprising. However, Borlotti et al [20] astutely pointed out that not all 
of the energy of the reflected wave is expected to return to the ascending aorta, because the junction of the ascending aorta with its daughter branches is well-matched in the forward but not backward direction. Based on the measured characteristic impedances of the vessels forming this junction, as reported by Cox and Pace [21], Borlotti et al calculated that the reflection coefficient at the aortic arch junction is 0.02 for a wave travelling forwards into the descending aorta, but -0.48 for a wave travelling backwards into the ascending aorta. While these coefficients in fact relate to pressure, not wave intensity, this nevertheless implies that not all of the reflected wave energy from the occlusion site propagates back into the aorta; some of it is re-reflected and some is transmitted into the supra-aortic vessels. Borlotti et al state that "this may be the reason for the observation of small backward waves at the aortic root, even during the occlusion, using the reservoirwave model", and conclude that "in the absence of other independent techniques or evidence, it is not currently possible to decide which [reflection index] is more correct" [20].

In this instance, however, a gold-standard reflection index is available. While Borlotti et al calculated pressure reflection coefficients, the same underlying theory may be used to calculate wave intensity reflection and transmission coefficients (see Table 3.1 in [22]). As shown in the appendix, by employing only the characteristic impedance data reported by Cox and Pace [21], and applying a simple wave tracking procedure, a gold-standard reflection index of 0.28 can be computed, although if second generation re-reflections are accounted for a value of 0.22 may be obtained (see appendix). These values are remarkably close to the average measured value of 0.25 given in Table 2 of Borlotti et al for conventional wave analysis and contrasts starkly with the reservoir-based value of 0.07 [20]. This therefore constitutes strong evidence from an independent technique using the same 
in vivo data from Borlotti et al that confirms the accuracy of conventional wave analysis and the inaccuracy of the reservoir-wave model.

It is a well-known phenomenon in science that new models are initially resisted, perhaps out of a desire to preserve the status quo. On the other hand, new models should not be accepted simply because they are new. Rather, a new model should be properly scrutinized from all angles before being adopted. In the case of the reservoir-wave model, numerous problems have been identified on the basis of theoretical considerations and numerical validation studies $[11-13,18]$. A more complete model that is not susceptible to these problems has recently been described, and addresses the perceived issues that the reservoir-wave model was designed to overcome [15]. In addition, comparison of in vivo data with a gold standard reference method has invalidated a key assertion of the reservoirwave model, i.e. that reservoir-based wave analysis is more accurate than conventional analysis. Combining all of these considerations, we suggest that the tide of the reservoirwave model may be ebbing.

\section{Appendix}

The experimental setup described by Borlotti et al [20] is illustrated in Figure 1, including the occluded descending thoracic aorta (DTA). At the onset of ventricular ejection, a forwardrunning compression wave (FCW, red arrows in Figure 1) propagates up the ascending aorta (AAo) and is transmitted into the DTA, brachiocephalic trunk (BCT) and subclavian artery (SC). General expressions for reflection and transmission coefficients for pressure, velocity and wave intensity have been derived in [22]. In this instance, the transmission coefficient for the pressure wave $(p)$ passing from the AAo into the DTA is 


$$
T_{p}^{A A o \rightarrow D T A}=\frac{2 Y_{A A o}}{Y_{A A o}+Y_{D T A}+Y_{B C T}+Y_{S C}}
$$

where $Y$ refers to the characteristic admittance of the respective vessels (the inverse of characteristic impedance). The transmission coefficient for a velocity wave $(u)$ passing from the AAo into the DTA is

$$
T_{u}^{A A O \rightarrow D T A}=\frac{2 Y_{D T A}}{Y_{A A O}+Y_{D T A}+Y_{B C T}+Y_{S C}}\left(\frac{A_{A A O}}{A_{D T A}}\right)
$$

where $A_{A A O}$ and $A_{D T A}$ are the respective cross-sectional areas of these vessels. Wave intensity is defined as $w i=d p d u$ and hence the AAo to DTA wave intensity transmission coefficient can be found by multiplying the pressure and velocity coefficients as follows,

$$
T_{w i}^{A A o \rightarrow D T A}=T_{u}^{A A 0 \rightarrow D T A} T_{p}^{A A 0 \rightarrow D T A}=\frac{4 Y_{A A 0} Y_{D T A}}{\left(Y_{A A o}+Y_{D T A}+Y_{B C T}+Y_{S C}\right)^{2}}\left(\frac{A_{A A O}}{A_{D T A}}\right)
$$

Similarly, it can be shown that the wave intensity transmission coefficient for a backward wave passing from the DTA to AAo is

$$
T_{w i}^{D T A \rightarrow A A O}=\frac{4 Y_{A A O} Y_{D T A}}{\left(Y_{A A O}+Y_{D T A}+Y_{B C T}+Y_{S C}\right)^{2}}\left(\frac{A_{D T A}}{A_{A A o}}\right)
$$

If there is a discrete reflection site in the DTA characterised by a reflection coefficient $R_{D T A}$, then the expected reflection index (defined by Borlotti et al [20] as the ratio of backward to forward peak wave intensities) in the AAo can be predicted using a wave tracking principle, as follows,

$$
R l_{A A o}=\frac{d l_{B C W}}{d l_{F C W}}=T_{w i}^{A A O \rightarrow D T A} R_{D T A} T_{w i}^{D T A \rightarrow A A o}=R_{D T A} \frac{16 Y_{A A O}^{2} Y_{D T A}^{2}}{\left(Y_{A A o}+Y_{D T A}+Y_{B C T}+Y_{S C}\right)^{4}}
$$


Referring to Figure 1, this envisages the AAo forward compression wave being transmitted into the DTA ( $\left.T_{w i}^{A A O \rightarrow D T A}\right)$, wave reflection occurring at the occlusion site $\left(R_{D T A}\right)$ giving rise to a backward compression wave (BCW, blue arrows in Figure 1) that is then transmitted back into the AAo $\left(T_{w i}^{D T A \rightarrow A A o}\right)$. Obtaining characteristic admittances from Cox and Pace [21], as in Borlotti et al [20], and assuming that the DTA occlusion was complete (i.e. $R_{D T A}=1$ ), the predicted AAo reflection index is 0.28 .

We can also account for re-reflections using the same wave tracking algorithm. The BCW in the DTA is not only transmitted into the AAo, but is also partially reflected back towards the occlusion site (green arrow in Figure 1). Since the reflection coefficient is negative in this direction, the resultant wave is a forward expansion wave. This wave is again reflected completely at the occlusion site, producing a backward expansion wave that is transmitted into the AAo (purple arrows) and would tend to subtract from the BCW (blue arrows) if these two waves overlapped in time. Extending the calculations to include this second generation reflection leads to a reflection index of 0.22 . Further generations of wave reflection have little effect on the predicted reflection index.

\section{Acknowledgments}

J. P. Mynard acknowledges the support of a CJ Martin Early Career Fellowship from the National Health and Medical Research Council of Australia. 


\section{References}

1. Sagawa K, Lie RK, Schaefer J. Translation of Otto Frank's paper "Die grundform des arteriellen pulses" Zeitschrift fur Biologie 37: 483-526 (1899). J Mol Cell Cardiol. 1990; 22 (3):253-277.

2. Westerhof N, Elzinga G, Sipkema P. An artificial arterial system for pumping hearts. J Appl Physiol. 1971; 31 (5):776-781.

3. Womersley JR. An elastic tube theory of pulse transmission and oscillatory flow in mammalian arteries: WADC Technical Report; 1957. Report No. TR 56-614.

4. Mitchell GF, Hwang S-J, Vasan RS, Larson MG, Pencina MJ, Hamburg NM, et al. Arterial stiffness and cardiovascular events: The Framingham heart study. Circulation. 2010; 121 (4):505-511.

5. Blacher J, Asmar R, Djane S, London GM, Safar ME. Aortic pulse wave velocity as a marker of cardiovascular risk in hypertensive patients. Hypertension. 1999; 33 (5):11111117.

6. McEniery CM, Yasmin, Hall IR, Qasem A, Wilkinson IB, Cockcroft JR. Normal vascular aging: Differential effects on wave reflection and aortic pulse wave velocity: The AngloCardiff Collaborative Trial (ACCT). J Am Coll Cardiol. 2005; 46 (9):1753-1760.

7. Torjesen AA, Wang N, Larson MG, Hamburg NM, Vita JA, Levy D, et al. Forward and backward wave morphology and central pressure augmentation in men and women in the Framingham heart study. Hypertension. 2014.

8. Weber T, Auer J, O'Rourke MF, Kvas E, Lassnig E, Berent R, et al. Arterial stiffness, wave reflections, and the risk of coronary artery disease. Circulation. 2004; 109 (2):184-189.

9. Weber T, Wassertheurer S, Rammer M, Haiden A, Hametner B, Eber B. Wave reflections, assessed with a novel method for pulse wave separation, are associated with end-organ damage and clinical outcomes / novelty and significance. Hypertension. 2012; 60 (2):534-541.

10. Wang JJ, O'Brien AB, Shrive NG, Parker KH, Tyberg JV. Time-domain representation of ventricular-arterial coupling as a windkessel and wave system. Am J Physiol Heart Circ Physiol. 2003; 284 (4):H1358-H1368.

11. Segers P, Taelman L, Degroote J, Bols J, Vierendeels J. The aortic reservoir-wave as a paradigm for arterial haemodynamics: Insights from three-dimensional fluid-structure interaction simulations in a model of aortic coarctation. J Hypertens. 2015; Publish Ahead of Print:10.1097/HJH.0000000000000449.

12. Mynard JP, Penny DJ, Davidson MR, Smolich JJ. The reservoir-wave paradigm introduces error into arterial wave analysis: A computer modelling and in-vivo study. J Hypertens. 2012; 30 (4):734-743.

13. Mynard JP. Assessment of conceptual inconsistencies in the hybrid reservoir-wave model. Proc Ann Int Conf IEEE Eng Med Biol Soc; 2013 3-7 July 2013; Osaka, Japan; 2013. p. 213-216. 
14. Tyberg JV, Bouwmeester JC, Parker KH, Shrive NG, Wang Jr J. The case for the reservoir-wave approach. Int J Cardiol. 2014; 172 (2):299-306.

15. Mynard JP, Smolich JJ. Wave potential and the one-dimensional windkessel as a wave-based paradigm of diastolic arterial hemodynamics. Am J Physiol Heart Circ Physiol. 2014; 307 (3):H307-H318.

16. Alastruey J. On the mechanics underlying the reservoir-excess separation in systemic arteries and their implications for pulse wave analysis. Cardiovasc Eng. 2010; 10 (4):176-189.

17. Hametner B, Wassertheurer S, Hughes AD, Parker KH, Weber T, Eber B. Reservoir and excess pressures predict cardiovascular events in high-risk patients. Int J Cardiol. 2014; 171 (1):31-36.

18. Mynard JP, Smolich JJ. The case against the reservoir-wave approach. Int J Cardiol. 2014; 176 (3):1009-1012.

19. Tyberg JV, Bouwmeester JC, Parker KH, Shrive NG, Wang J-J. Response to the letter of Mynard and Smolich. Int J Cardiol. 2014; 176 (3):1391.

20. Borlotti A, Park C, Parker KH, Khir AW. Reservoir and reservoir-less pressure effects on arterial waves in the canine aorta. J Hypertens. 2014; DOI: 10.1097/HJH.0000000000000425.

21. Cox RH, Pace JB. Pressure-flow relations in the vessels of the canine aortic arch. Am J Physiol. 1975; 228 (1):1-10.

22. Mynard JP. Computer modelling and wave intensity analysis of perinatal cardiovascular function and dysfunction [PhD Thesis]. Melbourne, Australia: University of Melbourne 2011. 
Figure 1 - Illustration of waves in the canine aortic arch upon occlusion of the descending thoracic aorta (DTA) as performed in the paper by Borlotti et al [20]. The initial forward compression wave (FCW) is transmitted from the ascending aorta (AAo) into the brachiocephalic trunk $(\mathrm{BCT})$, subclavian artery $(\mathrm{SC})$ and DTA, as shown with red arrows. This wave is reflected completely at the occlusion site, with the resulting backward compression wave (BCW) being partially transmitted back into the AAo, BCT and SC (blue arrows). However, the $\mathrm{BCW}$ is also partially reflected at the junction, giving rise to a second generation of reflected waves (green and purple arrows).

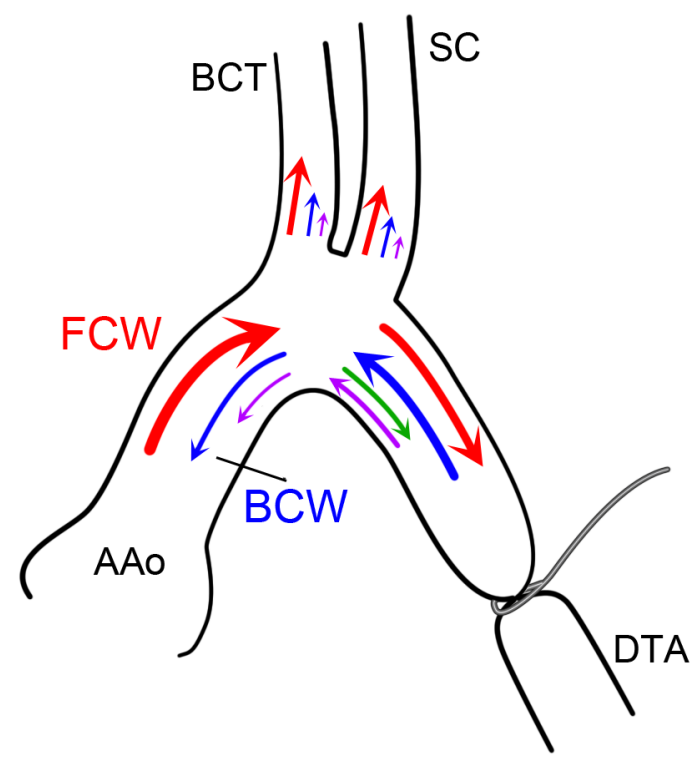




\section{University Library}

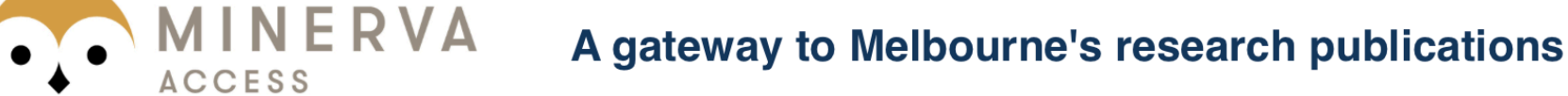

Minerva Access is the Institutional Repository of The University of Melbourne

Author/s:

Mynard, JP;Smolich, JJ;Avolio, A

Title:

The ebbing tide of the reservoir-wave model

Date:

2015-03-01

Citation:

Mynard, J. P., Smolich, J. J. \& Avolio, A. (2015). The ebbing tide of the reservoir-wave model. JOURNAL OF HYPERTENSION, 33 (3), pp.461-464. https://doi.org/10.1097/ HJH.0000000000000528.

Persistent Link:

http://hdl.handle.net/11343/124214 